\title{
Polymer-mediated immunocamouflage of red blood cells: Effects of polymer size on antigenic and immunogenic recognition of alloge- neic donor blood cells
}

\author{
WANG DunCheng ${ }^{1}$, Dana L. KYLUIK ${ }^{1,2}$, Kari L. MURAD ${ }^{3}$, \\ Wendy M. TOYOFUKU ${ }^{1} \&$ Mark D. SCOTT ${ }^{1,2^{*}}$ \\ ${ }^{1}$ Canadian Blood Services, Vancouver V6T 1Z3, British Columbia, Canada; \\ ${ }^{2}$ Centre for Blood Research and the Department of Pathology and Laboratory Medicine, \\ University of British Columbia, Vancouver V6T 1Z3, British Columbia, Canada; \\ ${ }^{3}$ Department of Biology, College of St. Rose, Albany 12208, New York, USA
}

Received December 1, 2010; accepted April 25, 2011; published online June 23, 2011

\begin{abstract}
Developing a practical means of reducing alloimmunization in chronically transfused patients would be of significant clinical benefit. Immunocamouflaging red blood cells (RBCs) by membrane grafting of methoxypoly(ethylene glycol) (mPEG) may reduce the risk of allo-immunization. The results of this study showed that antibody recognition of non-ABO antigens was significantly reduced in an mPEG-dose- and polymer size-dependent manner, with higher molecular weight mPEGs providing better immunoprotection. Furthermore, in vivo immunogenicity was significantly reduced in mice serially transfused with mPEG-modified xenogeneic (sheep; sRBCs), allogeneic (C57B1/6), or syngeneic (Balb/c) RBCs. Following a primary transfusion of sRBCs, mice receiving mPEG-sRBCs showed a $>90 \%$ reduction in anti-sRBC IgG antibody levels. After two transfusions, mice receiving $\mathrm{mPEG}$-sRBCs showed reductions of $>80 \%$ in anti-sRBC IgG levels. Importantly, mPEG-modified autologous cells did not induce neoantigens or an immune (IgG or IgM) response. These data suggest that the global immunocamouflage of RBCs by polymer grafting may provide a safe and cost-effective means of reducing the risk of alloimmunization.
\end{abstract}

methoxypoly(ethylene glycol), erythrocyte, blood group antigens, mice, alloimmunization

Citation: Wang D C, Kyluik D L, Murad K L, et al. Polymer-mediated immunocamouflage of red blood cells: Effects of polymer size on antigenic and immunogenic recognition of allogeneic donor blood cells. Sci China Life Sci, 2011, 54: 589-598, doi: 10.1007/s11427-011-4190-x

Safe and effective blood transfusions are arguably one of the most important medical advances made in the past century. These procedures are now common place and have saved millions of lives; however, until recently, this process has been of only limited interest to the research community. There is a substantial body of evidence showing that clinical problems can occur in chronically transfused patients because of alloimmunization [1-3]. These problems are compounded by the fact that it is difficult, time-consuming and

*Corresponding author (email: mdscott@interchange.ubc.ca) expensive to phenotype blood cells for mismatched antigens at non- $\mathrm{ABO} / \mathrm{RhD}$ epitopes. Yet these mismatches can, and do, have a significant impact on patient care-especially for chronically transfused sickle cell and thalassemic patients. Indeed, by some estimates, up to $35 \%$ of sickle patients show clinically significant levels of alloimmunization (i.e., shortened RBC survival and mild fever) to non-ABO/RhD antigens $[4,5]$. Of these alloimmunized patients, some become so reactive to donor RBCs that even the most closely matched samples cause a transfusion reaction, thus endangering the life of the patient. 
To address the problem of donor RBC alloimmunization, several clinical approaches have been suggested. These interventions range from minimal preventative action (addressing the problems of alloimmunization only after it occurs) to extensive prescreening of donor and recipient blood for the antigens most commonly associated with an increased risk of alloimmunization [6,7]. This latter approach has been criticized because the screening process is expensive and alloimmunization typically requires repeated antigen exposure [8]. While this argument has economic merit, it is akin to not screening donor blood for transmissible disease and only treating the patient after they have been infected. Therefore, the development of a cost-effective and practical means of reducing the risk of alloimmunization to all/most RBC alloantigens would be of significant clinical benefit.

Ongoing work on the covalent modification of RBC and other blood cells with methoxypoly(ethylene glycol) (mPEG; PEGylation) suggests that polymer grafting may prove to be a practical prophylactic method of decreasing the risk of transfusional alloimmunization and a treatment for patients that have already been alloimmunized [9-20]. To further examine the potential clinical application of "immunocamouflaged" erythrocytes, the effects of polymer size on the antigenic recognition of non-ABO blood group antigens expressed by normal human RBCs was investigated. Also, the immunocamouflage and immunogenic potential of unmodified and PEGylated syngeneic, allogeneic and xenogeneic RBCs were examined in a murine transfusion model.

\section{Materials and methods}

\subsection{RBCs and human sera}

After obtaining informed consent, whole venous blood was drawn from normal healthy volunteers into heparin-containing Vacutainer tubes. To prevent storage artifacts, all samples were processed immediately. Care was taken to ensure adequate representation of males and females, and no individuals were excluded on the basis of race or gender. Erythrocytes were washed three times in isotonic saline prior to use. Serum was prepared from venous blood collected in serum separator tubes (SST Vacutainer tubes; Becton Dickinson, Fairview, NJ, USA) and stored at $-70^{\circ} \mathrm{C}$. All biochemicals (unless stated otherwise) were obtained from Sigma Chemical Co. (St. Louis, MO, USA).

\subsection{Preparation of PEG-modified RBCs}

To assess the effect of molecular weight on the immunocamouflage of blood group antigens and intact RBCs, monofunctional (i.e., one binding site per chain) activated mPEGs of 2-30 kD were examined. Cyanuric chloride-activated
mPEG (C-mPEG; $5 \mathrm{kD}$ ) and unactivated, soluble, mPEG (sol-mPEG; $5 \mathrm{kD}$ ) were obtained from Sigma. Methoxypoly(ethylene glycol)-benzotriazolyl carbonate (BTC-mPEG; 5 and $20 \mathrm{kD}$ ) was obtained from Nektar Therapeutics (San Carlos, CA, USA). Succinimidyl valerate methoxypoly(ethylene glycol) (SVA-mPEG; 2, 5, 20 and $30 \mathrm{kD}$ ) was obtained from Laysan Bio Inc. (Arab, AL, USA). In addition, fluorescein-SVA-mPEG (fluor-SVA-mPEG; Laysan Bio Inc.) was used to visualize the homogeneity of polymer grafting.

Washed human erythrocytes were covalently grafted with activated mPEGs as previously described [9-11,14]. Stock solutions of PEG-derivatives were made in PEG-buffer (50 mmol L ${ }^{-1}$ dibasic potassium phosphate, $105 \mathrm{mmol} \mathrm{L}^{-1}$ $\mathrm{NaCl}, \mathrm{pH}$ 8.0) and mixed with washed human erythrocytes (12\% final hematocrit) using a micromixing device to assure homogeneous modification. Derivatization reactions were carried out at room temperature for $30 \mathrm{~min}$ or, in some cases, $60 \mathrm{~min}$. Modified RBCs were then washed three times with phosphate buffered saline (PBS). A control group comprising RBC incubated in mPEG-free PEG-buffer (0 mmol L ${ }^{-1}$ mPEG-RBC) was used to determine the effects of blood processing on immune complex binding. RBCs incubated with sol-mPEG (i.e., incapable of membrane grafting) were included as an additional control.

\subsection{Two-phase partitioning}

Partitioning of control and PEG-modified RBCs was carried out in an aqueous two-phase system comprising PEG8000 (Sigma) and Dextran T500 (molecular weight 500000; Sigma) as previously described [13]. The concentrations of the PEG and Dextran stock solutions were determined by refractive index measurements. Control or modified RBCs were added to $1 \mathrm{~mL}$ of PEG8000 and mixed. An aliquot $(0.5 \mathrm{~mL})$ of the PEG-RBC mixture was then overlaid on $0.5 \mathrm{~mL}$ of Dextran T500 and mixed. The two-phase system was allowed to separate at room temperature for $20 \mathrm{~min}$ prior to quantitation of the RBCs (via hemoglobin concentration) in the PEG layer. The results were expressed as the percentage of total hemoglobin in the upper PEG phase and were representative of a minimum of three independent experiments.

\subsection{Anti-A/B antibody mediated RBC agglutination}

The effect of grafted polymers on anti-sera-mediated RBC agglutination was assessed using a modified platelet aggregometer assay as previously described $[9,10]$. This assay allows the highly reproducible quantitation of the effects of PEGylation on anti-sera-mediated agglutination. Briefly, $450 \mu \mathrm{L}$ of control or PEGylated RBC (blood type A) suspension ( $6 \%$ hematocrit in isotonic saline) were placed in an aggregometer cuvette at $37^{\circ} \mathrm{C}$ with stirring. Anti-A typing serum (or pooled human antiserum; $20 \mu \mathrm{L}$ ) was added and 
RBC aggregation was monitored over time.

\subsection{Detection of blood group antigens by flow cytome- try}

Blood typing antibodies, unless otherwise noted, were purchased from Immucor, Inc. (Norcross, GA, USA). To quantitate the degree of immunocamouflage induced by the grafted polymers, flow cytometry (FACSCalibur; BD Biosciences San Jose, CA, USA) was performed using previously established erythrocyte parameters [12-14]. A minimum of 20000 cells was analyzed per sample. Control and PEG-modified RBCs were diluted to a $3 \%$ hematocrit in isotonic saline and resuspended in an equal volume of a specific anti-RBC antibody. The RBC-antibody mixtures were then incubated for $30 \mathrm{~min}$ at $37^{\circ} \mathrm{C}$ followed by washing twice with saline. A fluorescein isothiocyanate (FITC)conjugated anti-human IgG-Fc specific secondary antibody (Sigma; $50 \mu \mathrm{L}$; diluted 1:50 in saline) was added to each sample tube and the mixtures incubated at $37^{\circ} \mathrm{C}$ for $30 \mathrm{~min}$. The RBCs were washed twice in saline and fixed in $1 \%$ methanol-free formaldehyde (MFF). Immunocamouflage of blood group antigens was assessed using both Percent Gated and Mean Cell Fluorescence (MCF).

\subsection{In vivo $\mathrm{RBC}$ survival studies}

To examine the effects of the various $\mathrm{MPEG}$ derivatives in vivo, RBC survival studies were performed in Balb/c mice $[9,12,13]$. All animal studies were institutionally approved and conducted in compliance with all applicable Canadian animal care regulations. RBCs were collected from donor mice via cardiac puncture and derivatized with SVA-mPEG (20 kD) as previously described. The concentration of mPEG used for the in vivo studies ranged from 0 to $2 \mathrm{mmol} \mathrm{L}^{-1}$, since murine RBCs are physically smaller (i.e., have fewer surface binding sites for $\mathrm{mPEG}$ ) and more fragile than human RBCs. Murine RBCs were labeled using the fluorescent membrane anchored marker, PKH-26 (Sigma). Blood samples from recipient mice were monitored until the labeled RBC was cleared from the circulation (approximately $50 \mathrm{~d}$ for unmodified syngeneic cells). Survival of fluorescently labeled mPEG-treated and control RBCs was monitored by analyzing the percentage of fluorescently-labeled RBCs in the peripheral circulation by flow cytometry. A minimum of five mice was used per mPEG concentration tested.

\section{7 vivo $\mathrm{RBC}$ immunogenicity}

For in vivo immunogenicity studies, two genetically distinct mouse strains (C57B16 and Balb/c; $\mathrm{H}-2 \mathrm{~K}^{\mathrm{b}}$ and $\mathrm{H}-2 \mathrm{~K}^{\mathrm{d}}$ ) were used to assess alloimmunization $[21,22]$. Donor RBCs were obtained from C57/Black or Balb/c mice by cardiac puncture. To further assess the immunomodulatory effect of
PEGylation, sheep RBCs were obtained from Cocalico (Reamstown, PA, USA). Mouse and sheep RBCs were washed three times in isotonic saline, and resuspended at a final concentration of $5 \mathrm{~g} \%(\sim 12 \%$ hematocrit). The RBC suspension was derivatized with $\mathrm{C}-\mathrm{mPEG}$ at concentrations of $0,1.2$ and $5 \mathrm{mmol} \mathrm{L}^{-1}$ for $30 \mathrm{~min}$ at $4^{\circ} \mathrm{C}$ in PBS (pH 9.2). After derivatization, the cells were washed three times in isotonic saline and resuspended to a $50 \%$ hematocrit prior to transfusion.

Recipient mice (Balb/c; $n=24)$ were transfused intraperitoneally (i.p.) with $500 \mu \mathrm{L}$ of control or mPEG-modified $\mathrm{C} 57 \mathrm{~B} 1 / 6$ or Balb/c RBCs. This procedure was repeated for up to three transfusions (each at $\sim 50 \mathrm{~d}$ intervals). At the end of each transfusion, eight mice per group were sacrificed and serum was collected for analysis of anti-donor antibody formation. Sera from four mice were pooled, while the remaining four mice were analyzed separately (to assess response variation) for $\operatorname{IgG}$ and $\operatorname{IgM}$ antibodies against the transfused RBCs. Antibody binding was quantitated by mixing $600 \mu \mathrm{L}$ of serum with saline (at ratios of $2: 1,1: 1$, and 1:5) with $200 \mu \mathrm{L}$ of an $80 \%$ hematocrit of unmodified donor RBCs. The samples were mixed for $30 \mathrm{~min}$ at $37^{\circ} \mathrm{C}$ and then washed three times in isotonic saline. Secondary antibodies to mouse IgG (PE labeled) and IgM (FITC-labeled) were added to $20 \mu \mathrm{L}$ of the previously incubated packed $\mathrm{RBCs}$ and mixed for $30 \mathrm{~min}$ at $37^{\circ} \mathrm{C}$ followed by washing three times in isotonic saline. Secondary antibody binding was quantitated by flow cytometry as described above.

The immunogenicity of control and PEGylated xenogeneic sheep RBC was similarly assessed in Balb/c mice. Mouse serum was collected $14 \mathrm{~d}$ after the primary transfusion of sheep RBCs and the presence of mouse anti-sheep RBC IgG and IgM was assessed by flow cytometry. In addition, some mice received a second transfusion of sheep cells ( $14 \mathrm{~d}$ after the primary transfusion) and serum was collected 28 days post-primary transfusion and assessed for anti-sheep IgG and IgM. Mice were transfused with a blood volume approximating $10 \%$ of the total mouse blood volume and each group contained a minimum of eight mice.

\subsection{Statistical analysis}

All results were expressed as the mean \pm standard error of the mean (SEM) unless otherwise stated. Each experiment was repeated a minimum of three times although, in some cases (e.g., microscopy), representative samples are presented. Statistical analysis was performed using SPSS v.16.0 statistical software (SPSS Inc., Chicago, IL, USA). One-way analysis of variance (ANOVA) followed by Tukey's posthoc test was performed for comparison of three or more mean values. An independent variable $t$-test was used for comparison of two mean values. A two-tailed $P$-value $<0.05$ was considered significant. 


\section{Results}

Biophysically, membrane grafted polymers alter the surface characteristics of cells as denoted by changes in separation profiles in a two-phase (Dextran-PEG) partitioning system. As shown in Figure 1A, the biophysical partitioning of modified RBCs is dependent upon both grafting concentration and polymer size. However, large polymers more effectively shifted the modified RBCs into the PEG layer, even at very low grafting concentrations. As shown, grafting of the $2 \mathrm{kD}$ polymer only resulted in significant partitioning of the RBCs to the PEG layer at higher grafting concentrations. By contrast, the 20 and $30 \mathrm{kD} \mathrm{SVA-mPEG} \mathrm{modified} \mathrm{RBCs}$ rapidly moved into the PEG layer, even at very low grafting concentrations, with maximal partitioning occurring at $\geqslant 0.5 \mathrm{mmol} \mathrm{L}^{-1}$. Moreover, even at relatively modest grafting concentrations ( $1 \mathrm{mmol} \mathrm{L}^{-1}$ SVA-mPEG-20 kD) RBCs were uniformly derivatized, as shown by overlay of light and fluorescent photomicrographs of the SVA-mPEG-RBC relative to the control cells (Figure 1B). Importantly, linker chemistry (as shown by the $5 \mathrm{kD} \mathrm{C}$ - and SVA-mPEG) was not a significant factor in phase separation as long as the linker chemistries showed similar reactivity and hydrolysis rates.

Polymer length was also important in preventing antigenic recognition of the $\mathrm{A} / \mathrm{B}$ antigens on RBCs. As shown in Figure 2, polymer lengths of 2, 5 and $20 \mathrm{kD}$ all conferred some degree of protection against antisera-mediated agglutination of type A RBCs. This protection against antibody- mediated agglutination was both size- and dose-dependent, with long chain polymers providing significantly better inhibition of agglutination (Figure 2A-C). This finding correlates well with the observed partitioning of RBCs in the PEG-Dextran two-phase system. As noted in Figure 2C, grafting concentrations $\geqslant 1.2 \mathrm{mmol} \mathrm{L}^{-1}$ of the $20 \mathrm{kD}$ polymer resulted in the complete loss of anti-A-mediated agglutination. Grafting efficacy was also highly malleable in terms of both $\mathrm{pH}$ and the grafting time of the PEGylation reaction (Figure 2D). No statistically significant difference was observed between $\mathrm{pH} 8.0$ and 9.2 after either 30 or $60 \mathrm{~min}$ of derivatization $\left(2.5 \mathrm{mmol} \mathrm{L}{ }^{-1} 5 \mathrm{kD}\right.$ SPA-mPEG). Similarly, while a 60-min grafting reaction appeared to confer some advantages in terms of inhibiting anti-A agglutination, this observed difference ( $2.5 \mathrm{mmol} \mathrm{L}^{-1} 5 \mathrm{kD}$ SPA-mPEG) was not significant when the effect of time was assessed using the control cells ( $\left.0 \mathrm{mmol} \mathrm{L}^{-1} \mathrm{SPA}-\mathrm{mPEG}\right)$.

While immunocamouflage of $\mathrm{A} / \mathrm{B}$ antigens was observed, it was not sufficient to yield a true "universal erythrocyte" as the method cannot remove all risk from an untyped blood recipient. However, immunocamouflage of the non-ABO blood group antigens holds much greater clinical potential. The Rhesus blood group antigens, especially $\mathrm{RhD}$, are particularly important in transfusion medicine. As shown in Figure 3, RhD, Rhc, and RhE are all effectively camouflaged by grafted mPEG. Immunocamouflage of the D, c, and $\mathrm{E}$ antigens is dependent upon both grafting concentration and polymer size. Immunocamouflage of antigenic epitopes is shown by the absolute camouflage (Percent
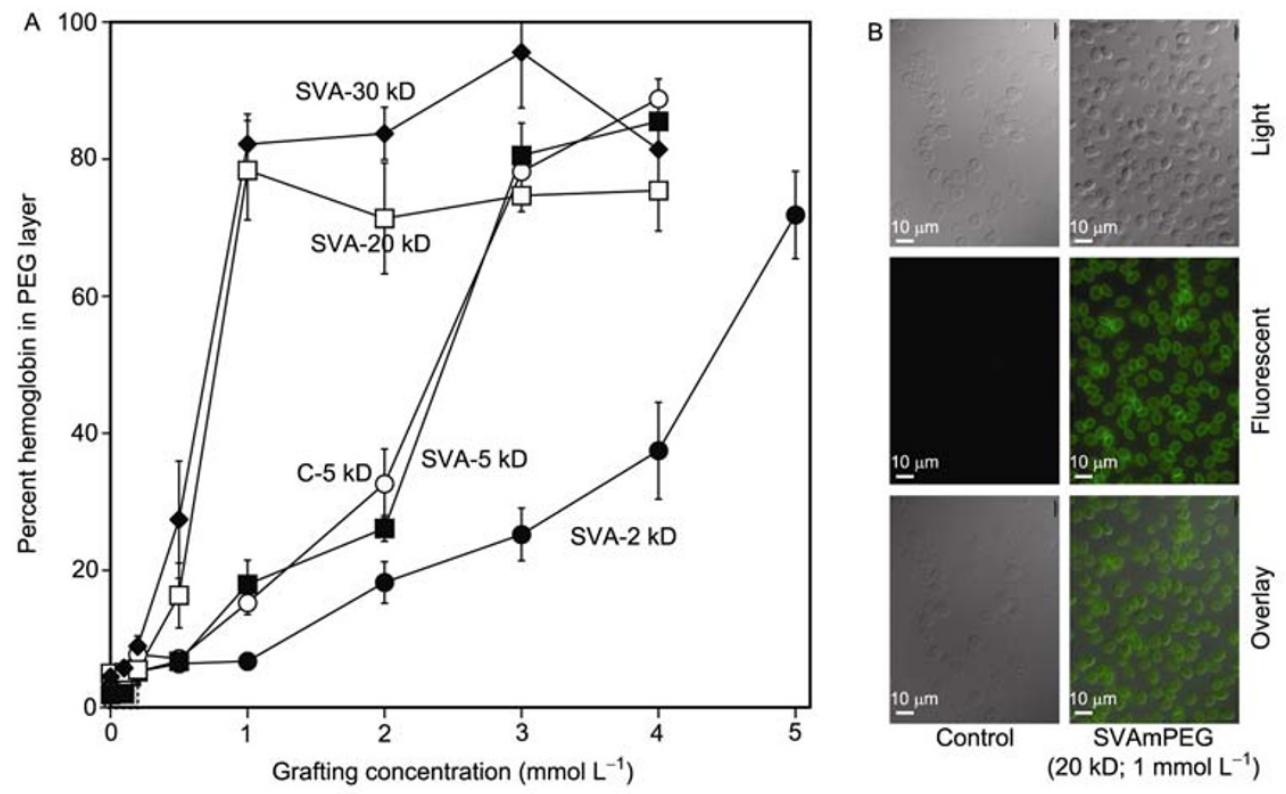

Figure 1 Polymer grafting results in size- and concentration-dependent phase separation. A, mPEG-RBCs preferentially partition to the PEG layer of a two-phase Dextran-PEG separation system. Larger polymers $(20$ and $30 \mathrm{kD})$ significantly $(P<0.001)$ enhance the separation compared with equimolar concentrations of small-chain $(2$ or $5 \mathrm{kD})$ polymers. Separation efficacy correlates well with the extent of immunocamouflage imparted by the grafted polymer. $\mathrm{B}$, Additionally, the grafted polymer provides uniform coverage of the modified RBCs, as shown by the grafted fluorescent SVA-mPEG (20 kD; 1 mmol $\mathrm{L}^{-1}$ ) to human RBC relative to the control RBCs. Differential interference contrast microscopy was used to assess the control and modified RBCs. Representative fields are shown. 

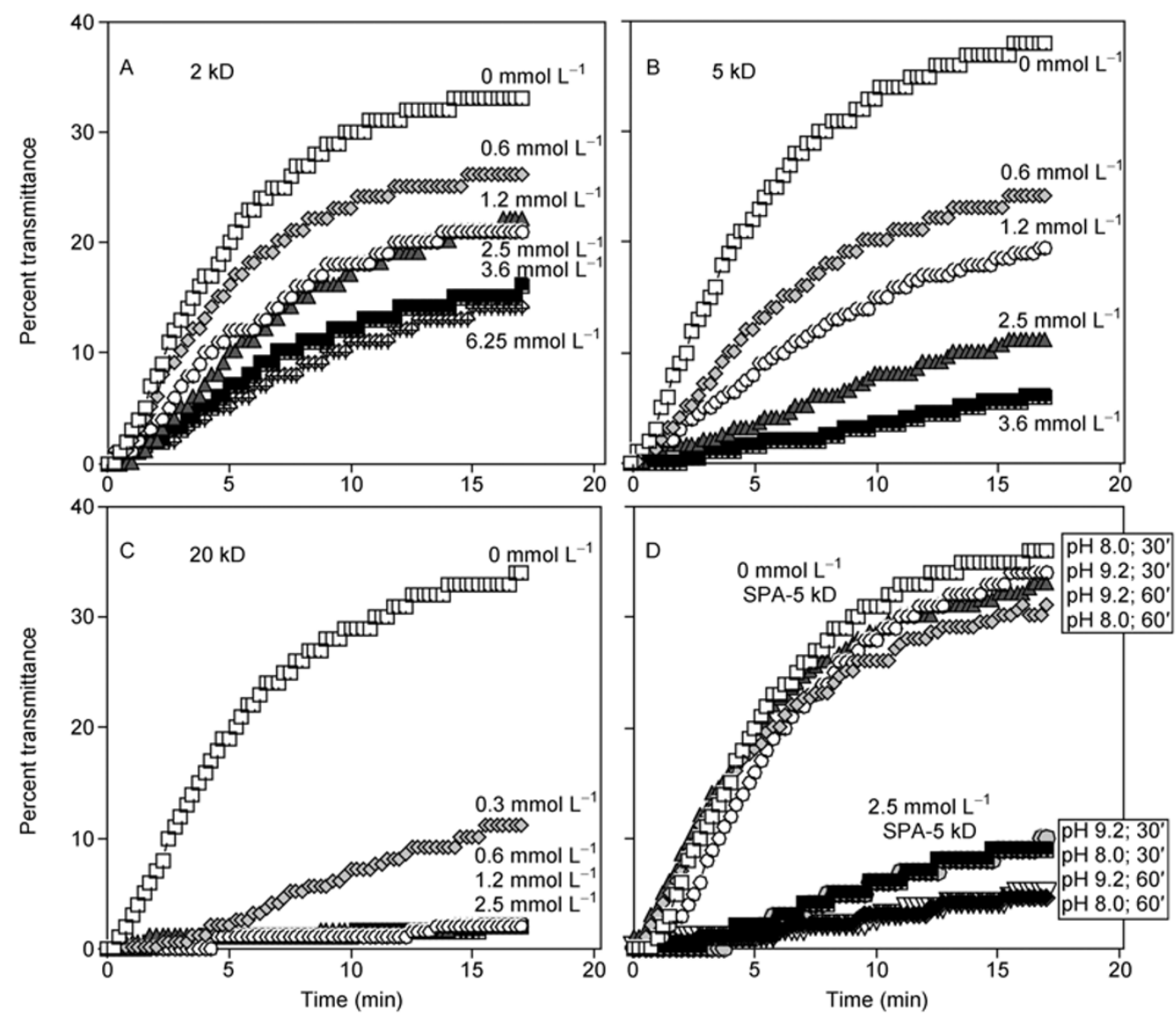

Figure 2 RBC agglutination is dramatically affected by both the chain length and grafting concentration of the mPEG polymer. Shown is a comparison of solution-phase agglutination studies using anti-A antisera and type A RBCs modified with different chain lengths of SPA-mPEG: 2 kD (A), 5 kD (B) and 20 $\mathrm{kD}(\mathrm{C})$. All sizes of SPA-mPEG polymers are able to decrease RBC agglutination. However, the molar concentration necessary to inhibit agglutination dramatically decreases as the polymer length increases. These three panels are representative of $>5$ independent assays. D, Changes in derivatization time and $\mathrm{pH}$ do not significantly impact polymer grafting as assessed by anti-A agglutination.

Positive Cells; Figure 3A-C) and the change in MCF (Figure $3 \mathrm{~A}$ and $\mathrm{B}$, inserts). As shown, polymer size is an important factor in the efficacy of immunocamouflage by the $20 \mathrm{kD}$ polymer, which showed significantly better efficacy in terms of percent antigen positive cells than the 2 $(P<0.001)$ or $5(P<0.01) \mathrm{kD}$ polymers at intermediate $(2-3$ mmol L $\left.{ }^{-1}\right)$ grafting concentrations. Relative to the percent positive RBCs, the MCF of modified cells was even more dramatically affected by the size of the grafted polymer. Compared with either the 2 or $5 \mathrm{kD}$ chains, the $20 \mathrm{kD}$ polymer very effectively camouflaged the bulk of $\mathrm{RhD}$ and Rhc antigens, as shown by the decrease in antibody binding. A decrease in the MCF would significantly impact the antigenic presentation efficacy of the donor cells. Importantly, as was noted in Figure 1, linker chemistry was not a significant factor, as both the $5 \mathrm{kD} \mathrm{C}$ and BTC-mPEGs and the $20 \mathrm{kD}$ SPA- and BTC-mPEGs yielded relatively comparable results. The small differences that were observed relative to linker chemistry are readily explained by the hydrolysis half-life of the linker chemistries (13.5 and $16.5 \mathrm{~min}$ for BTC- and SPA-mPEGs, respectively) and the 60-min deri- vatization time used in the experiments.

Other, non-ABO, blood group antigens from the Kidd $\left(\mathrm{Jk}^{\mathrm{a}}, \mathrm{Jk}^{\mathrm{b}}\right)$, Kell $(\mathrm{k})$ and Duffy $\left(\mathrm{Fy}^{\mathrm{a}}\right)$ families were also efficiently camouflaged by the grafted polymers. As shown in Figure $4, \mathrm{Jk}^{\mathrm{a}}, \mathrm{Jk}^{\mathrm{b}}, \mathrm{k}$, and $\mathrm{Fy}^{\mathrm{a}}$ were all camouflaged by the 2, 5 and $20 \mathrm{kD}$ grafted polymers. However, all polymer sizes were not equally effective at masking all blood group antigens. Of the blood groups shown in Figure 4, $\mathrm{k}$ was the most easily camouflaged-even by the $2 \mathrm{kD}$ polymer. By contrast, the $2 \mathrm{kD}$ polymer failed to immunocamouflage $\mathrm{Jk}^{\mathrm{b}}$ $(P>0.05)$ and provided only weak, though statistically significant $(P<0.05)$, protection to the $\mathrm{Jk}^{\mathrm{a}}$ blood group antigen. The $20 \mathrm{kD}$ polymer provided highly significant $(P<0.001)$ levels of protection for both $\mathrm{Jk}^{\mathrm{a}}$ and $\mathrm{Jk}^{\mathrm{b}}$, though it provided better protection for $\mathrm{Jk}^{\mathrm{a}}$. Hence, the nature of the antigen (height relative to the membrane surface) is a critical determinant in the choice of polymer.

While in vitro assays demonstrate the efficacy and potential clinical value of immunocamouflaged RBCs, in vivo efficacy and stability are both crucial for evaluating potential utility. Importantly, immunocamouflage of RBCs did 

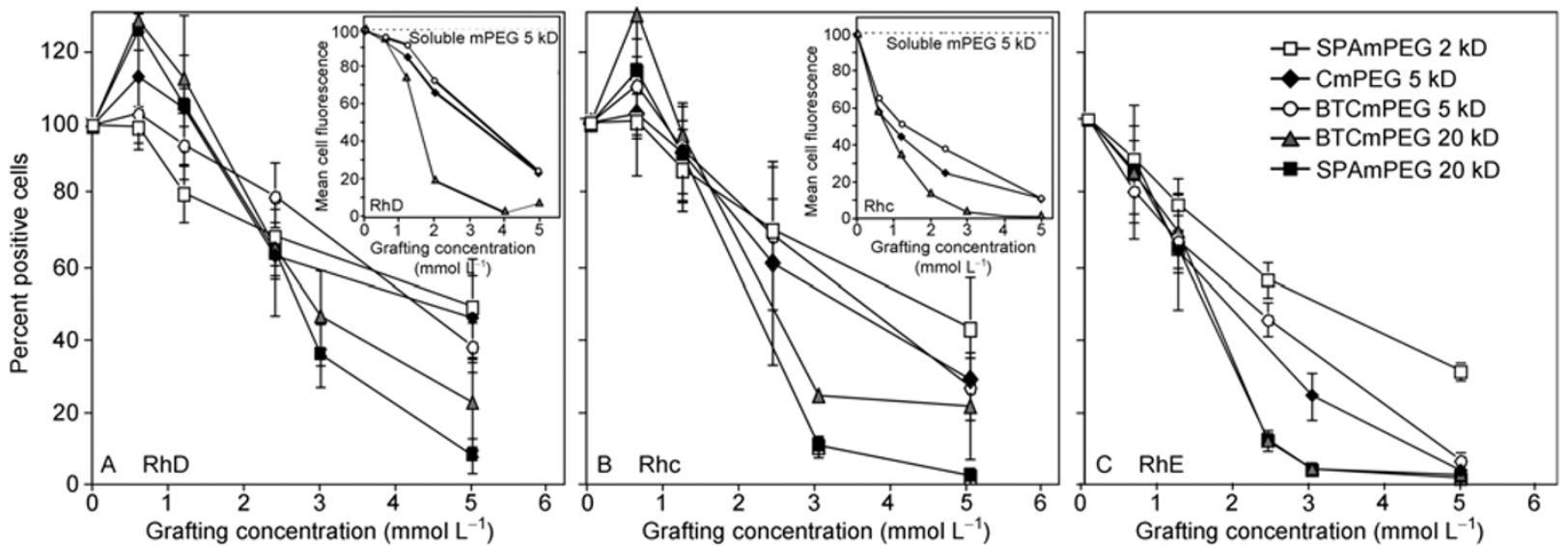

Figure 3 Rh blood group antigens are effectively immunocamouflaged by grafted polymers. Anti-RhD (A) and Anti-Rhc (B) and RhE (C) antibody binding is dramatically attenuated by mPEG-modification, as shown by a decrease in percent positive cells and MCF (A and B, inserts). The decrease in binding was a function of both grafting concentration $\left(0-5 \mathrm{mmol} \mathrm{L}^{-1}\right)$ and polymer size $(2,5$ or $20 \mathrm{kD})$. Importantly, as shown in the MCF inserts in A and B, unactivated mPEG (sol-mPEG) had no immunocamouflage effect (denoted by the labeled dashed line). Human RBCs were modified with $0-5$ mmol $\mathrm{L}^{-1}$ activated $\mathrm{mPEG}$ as described in Materials and methods. Results represent the mean $\pm \mathrm{SD}$ of a minimum of three independent experiments.
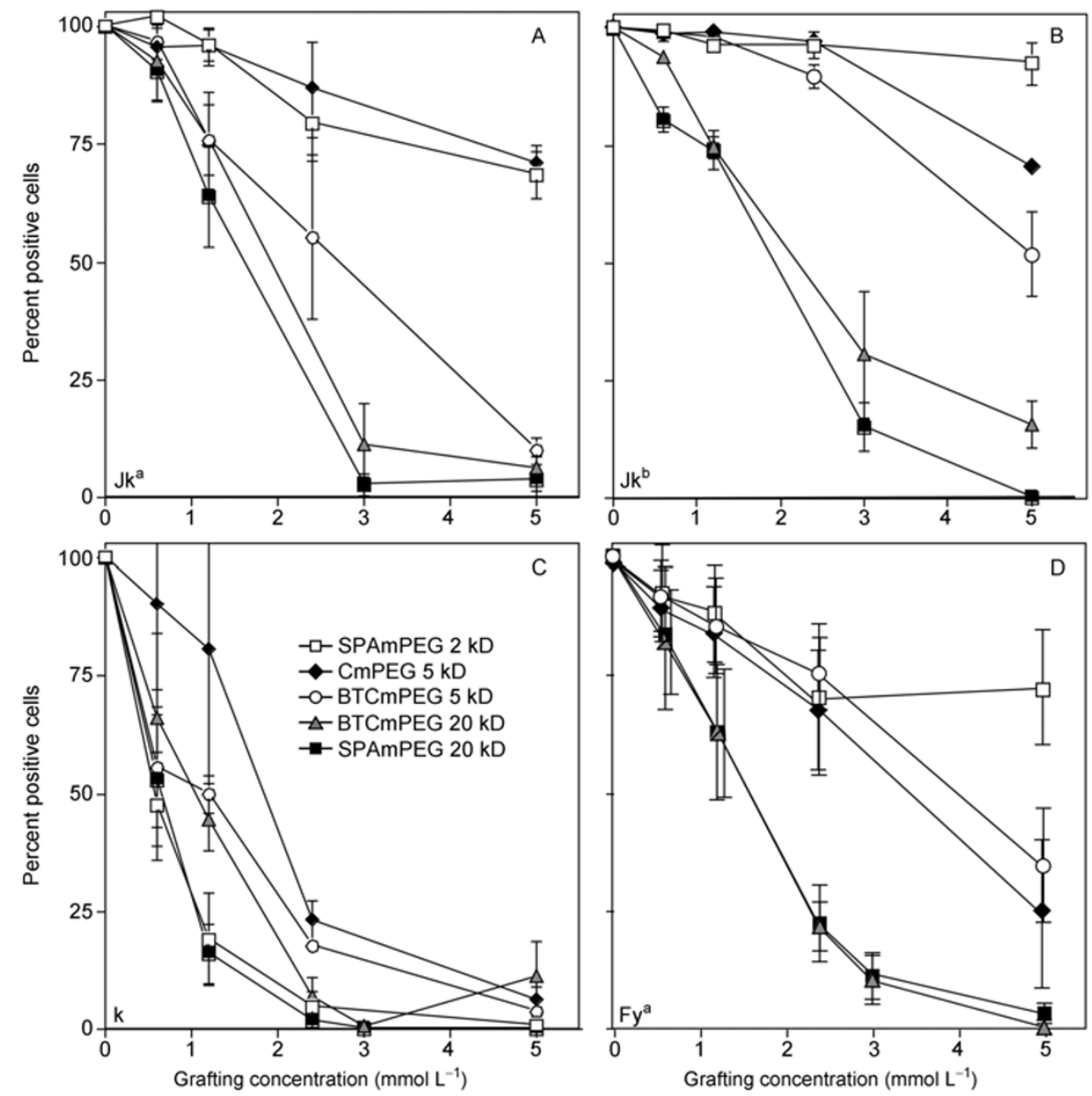

Figure 4 Derivatization of RBC with mPEG effectively attenuates antibody recognition of non-ABO antigens as shown on selected Kidd (Jka, Jkb; A and $\mathrm{B}$, respectively), Kell (k; C) and Duffy (Fya; D) antigens. The effects of 2, 5 and $20 \mathrm{kD}$ polymers on antibody binding to blood group positive human RBCs are shown. A minimum of 20000 counts per samples was analyzed by flow cytometry. The MCF of the antibody-positive cells also decreased dramatically for all blood group antigens (data not shown). 
not adversely affect in vivo survival in a murine transfusion model. Figure 5A shows both the mean clearance curves for PKH-26-labeled control and polymer-modified $\left(2 \mathrm{mmol} \mathrm{L}^{-1}\right.$ BTC-mPEG) RBCs and the clearance curves of the individual mice ( $n=5$ per group). As noted, no significant differences were observed between the groups and both populations fell within normal murine clearance patterns. Furthermore, Figure 5B shows that immunocamouflage of syngeneic donor cells did not induce the formation of neoantigens, or expose cryptoantigens, which would have resulted in immune recognition and clearance by the recipi- ent mice. This was demonstrated by a lack of IgG (shown) and IgM (not shown) antibody production to either the control or mPEG-RBCs over the course of 1-3 transfusions (up to $\sim 180 \mathrm{~d}$ ). More importantly, with regards to the potential clinical utility of immunocamouflaged RBC, polymer modified allogeneic (C57B1/6 into Balb/c) RBCs showed a highly significant $(P<0.001)$ blunting of antibody production relative to the unmodified allogeneic donor cells (Figure 5C). This effect remained potent after three transfusions with modified allogeneic donor RBCs $(\sim 180 \mathrm{~d})$. In contrast to mPEG-RBCs, unmodified allogeneic RBCs induced a sig-
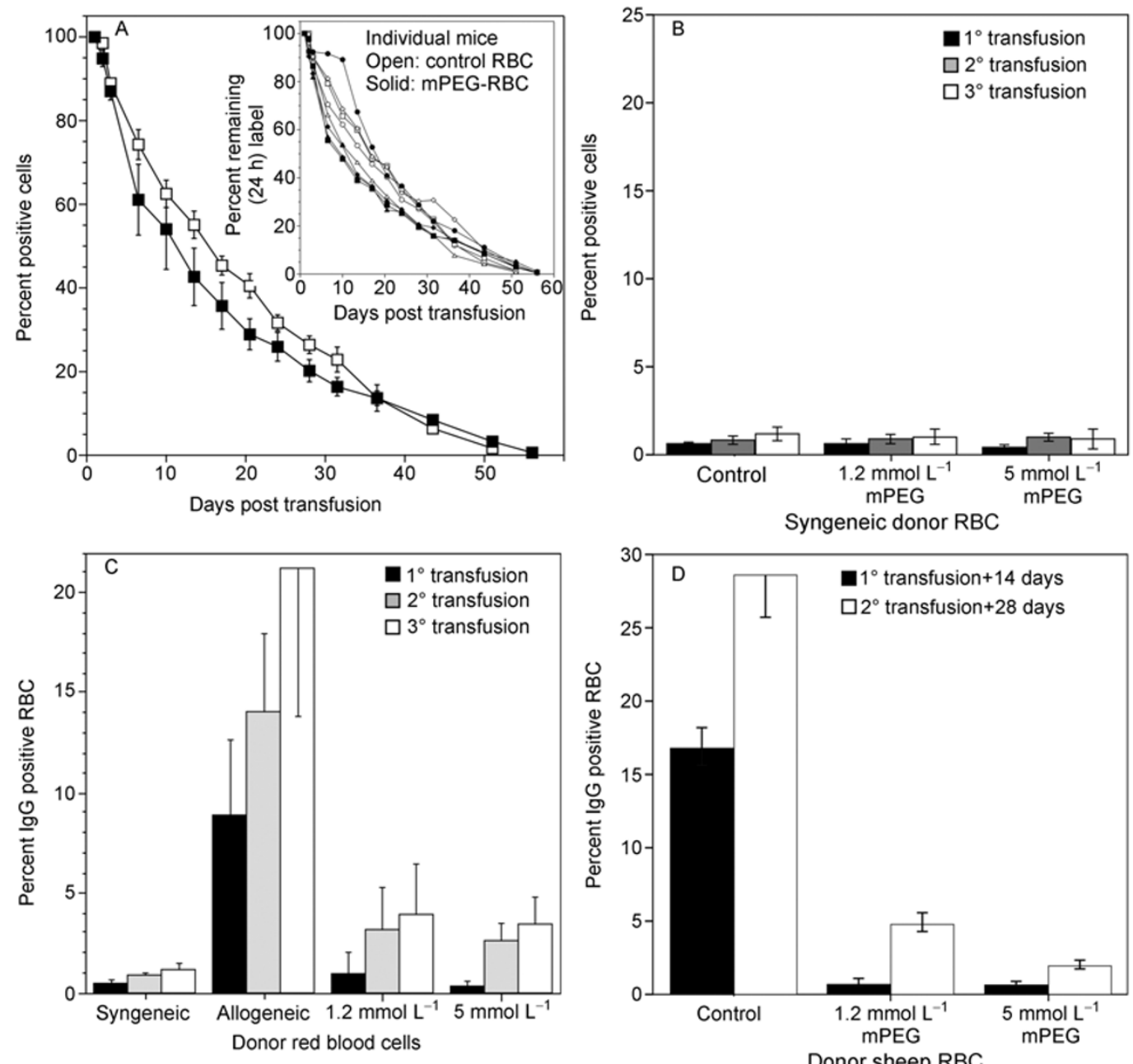

Figure 5 Effects of polymer modification of RBCs on in vivo survival and immunogenicity of syngeneic, allogeneic and xenogeneic donor cells. A, Syngeneic primary transfusions. The clearance rates after primary transfusion of control (open squares) and BTC-mPEG-derivatized (20 kD; solid squares) murine RBCs. PEGylated RBCs were tagged with a fluorescent fatty acid marker (PKH-26) and survival was followed by flow cytometry. The results represent the mean \pm SEM of five Balb/c mice per PEG concentration. The insert shows the clearance curves of each individual mouse. B, Neo-antigen formation was not observed upon transfusion with PEGylated RBCs. The percentage of IgG-positive RBCs, subsequent to 1-3 transfusions with control or PEGylated (1.2 or $5 \mathrm{mmol} \mathrm{L} \mathrm{L}^{-1} \mathrm{C}$-mPEG) syngeneic donor cells, is shown. Murine IgG-positive RBCs were detected by flow cytometry using a fluorescent-labeled anti-mouse IgG secondary antibody. C, The immunogenicity of allogeneic RBCs was significantly $(P<0.001)$ reduced by PEGylation of the donor cells, even after three challenges with the modified allogeneic donor cells. As shown, mice receiving unmodified donor cells produced significant amounts of IgG (but not IgM) in response to the allogeneic cells. This was not observed for the mPEG-RBCs. Recipient IgG (shown) and IgM (not shown) antibodies to the allogeneic cells were detected by flow cytometry. D, As an extreme test of the ability of immunocamouflage to prevent immunogenic recognition of donor cells, xenogeneic sheep RBCs (sRBCs) were PEGylated (1.2 or $5 \mathrm{mmol} \mathrm{L}{ }^{-1} \mathrm{C}$-mPEG). Following primary or secondary transfusion of mice with unmodified sRBCs, a potent IgG antibody response was noted 14 days post challenge. However, PEGylation of the donor sRBCs dramatically reduced $(P<0.001)$ the IgG response. This effect was maintained even after a second transfusion was administered on day 14 , and assessed on day 28 following primary exposure. A-D, Mice were transfused with a blood volume approximating $10 \%$ of the total mouse blood volume. Each group contained eight mice and the results shown represent the mean number of positive RBCs \pm SD. 
nificant antibody response, though it should be noted that these antibodies did not significantly affect donor cell circulation time. The immunocamouflaging effect of the grafted polymers also provided a potent $(P<0.001)$ immunoprotective effect on xenogeneic (sheep) RBCs (Figure 5D).

\section{Discussion}

Despite $\mathrm{ABO} / \mathrm{RhD}$ matching, the $\mathrm{RBC}$ remains an antigenically challenged cell. Adverse reactions, ranging from clinically insignificant to significant, arising from non-ABO/RhD antigens are relatively common, due in large part to varying expression of these epitopes by different ethnic and racial groups $[4,23]$. Indeed, transfusion reactions arising from the non- $\mathrm{ABO} / \mathrm{RhD}$ blood group antigens are most commonly encountered in minority populations. Coupled with this is the finding that the incidence of alloimmunization is also positively correlated with the number of transfusions an individual receives. Hence, alloimmunization to non-ABO/ $\mathrm{RhD}$ blood group antigens is exaggerated in certain individuals, especially minorities, with chronic diseases (e.g., sickle cell disease or thalassemia) receiving transfusion therapy $[1,4,24]$.

Consequent to alloimmunization, transfusion reactions against non- $\mathrm{ABO} / \mathrm{RhD} \mathrm{RBC}$ antigens can make it almost impossible to identify appropriate blood donors [25]. To prevent the risk of extreme alloimmunization, various clinical interventions have been explored. These include the now standard use of packed RBCs in place of whole blood (to prevent alloimmunization due to white blood cells), extensive pre-transfusion phenotype screening to more closely match donor and patient RBCs, and the long-term use of single RBC donors. Despite these often costly measures, clinically significant alloimmunization rates can still exceed $30 \%$ in patients with sickle cell anemia and thalassemia [26]. Consequently, many laboratories and clinicians question the cost effectiveness of donor screening as a prophylactic measure to prevent alloimmunization.

Hence, a cost effective means of diminishing/preventing the risk of alloimmunization is urgently needed. To this end, immunocamouflaged RBCs may prove to be of clinical value [20]. As shown in the present study, immunocamouflaged RBCs are characterized by global camouflage of the $\mathrm{ABO}$ and non-ABO human blood group antigens (Figures 2-4) and a significant attenuation of donor cell immunogenicity (Figure 5) in a murine transfusion model. This is effectively accomplished at dosages that do not adversely affect the in vitro or in vivo stability and function of human or murine RBCs. In addition, the procedure is inexpensive and can be readily adapted to existing blood banking technology.

While the moderate derivatization concentrations $(\leqslant 2.5$ mmol L ${ }^{-1} \mathrm{mPEG}$ per $0.6 \times 10^{9} \mathrm{RBC} \mathrm{mL}^{-1}$ ) that are likely to be used for human $\mathrm{RBC}$ do not completely camouflage the A/B blood group antigens, these grafting concentrations do result in significant masking of clinically relevant non-ABO antigens. Importantly, it is not necessary to achieve a state of complete immunocamouflage to significantly reduce the risk of alloimmunization due to these non-ABO blood groups. As shown in Table 1, the observed risk of alloimmunization is governed, in part, by the number of cells positive for a particular antigen, the apparent antigen density of the antigen on the cell, and the ability of appropriate antigen presenting cells to recognize, phagocytose and present that antigen. PEGylation of donor RBCs dramatically reduces the efficiency of each of these steps: The number of

Table 1 Antigenic abundance and relative immunogenicity of selected blood group antigens ${ }^{\mathrm{a}}$

\begin{tabular}{|c|c|c|c|c|c|}
\hline Blood group & Antigen & Percent immunogenicity $^{*}$ & Hemolytic transfusion reaction & Primary $\mathrm{Ab}$ & Copies per RBC \\
\hline $\mathrm{ABO}$ & A, B & $100^{\mathrm{Ac}}$ & Yes & IgM & $1 \times 10^{6}$ \\
\hline \multirow[t]{5}{*}{$\mathrm{Rh}$} & $\mathrm{D}$ & 50.00 & Yes & IgG & $10000-35000^{* *}$ \\
\hline & $\mathrm{c}$ & 2.05 & Yes & $\mathrm{IgG}$ & $37000-85000^{* *}$ \\
\hline & $\mathrm{E}$ & 1.69 & Yes & IgG & $450-25600^{* *}$ \\
\hline & $\mathrm{e}$ & 0.56 & Yes & $\operatorname{IgG}$ & $13400-24400^{* * *}$ \\
\hline & $\mathrm{C}$ & 0.11 & Yes & IgG & $42000-56000^{* * * *}$ \\
\hline \multirow[t]{2}{*}{ Kidd } & $\mathrm{Jk}^{\mathrm{a}}$ & 0.07 & Yes & IgG & $11000-14000^{\#}$ \\
\hline & $\mathrm{Jk}^{\mathrm{b}}$ & 0.03 & Yes & $\operatorname{IgG}$ & $11000-14000^{\#}$ \\
\hline Duffy & $\mathrm{Fy}^{\mathrm{a}}$ & 0.23 & Yes & IgG & $7000-13000^{\#}$ \\
\hline \multirow[t]{2}{*}{ Kell } & $\mathrm{k}$ & 1.50 & Yes & $\operatorname{IgG}$ & 3500 \\
\hline & K & 5.00 & Yes & $\operatorname{IgG}$ & 6000 \\
\hline \multirow[t]{2}{*}{ MNS } & S & 0.04 & Yes & $\operatorname{IgG}$ & $>12000$ \\
\hline & $\mathrm{s}$ & 0.03 & Yes & $\mathrm{IgG}$ & 12000 \\
\hline
\end{tabular}

a) Modified from Martin [27]. The antigenic sites are presented as ranges because the number expressed is dependent upon the absence or presence of other antigens. *, percentage of null recipients sensitized after a single transfusion. Ac, acute transfusion reaction. **, from Hughes-Jones et al. [28]; ***, from Masouredis et al. [29]; \#, from Skov [30]. 
antigen positive cells is reduced, the apparent antigen density on the positive cells is decreased, PEGylated cells are less effectively phagocytosed, and antigen presentation of PEGylated proteins is impaired [9,20-22,31].

The net result of these events on clinical practice may be illustrated by examining the potential effects that immunocamouflaged RBCs may have on the risk of alloimmunization to Rhc. Table 1 shows that 'clinical experiments' (i.e., unintentional mismatches at specific antigens) determine the relative risk of immunogenic recognition for Rhc to be approximately $2 \%$ after a single mismatch [28-30]. As noted in Figure 3, Rhc is readily camouflaged by mPEG-grafting, as shown by a decrease in both percent positive cells and MCF. This finding raises the question: What would be the residual alloimmunization risk of $\mathrm{Rhc}^{+}$donor RBCs subsequent to polymer grafting (e.g., $2.5 \mathrm{mmol} \mathrm{L}^{-1}$ of a $20 \mathrm{kD}$ polymer)? Our in vivo murine data (Figure 5) with allogeneic and xenogeneic donor cells suggests that the actual risk of alloimmunization would be significantly reduced. Hence, the $2 \%$ risk of immunogenic recognition may subsequently become a 0.2 or a $0.02 \%$ risk of alloimmunization. Thus, while some individuals will become alloimmunized, even after receiving PEGylated blood, the relative risk to an individual, and to the chronically transfused patient population as a whole, will be dramatically reduced.

Indeed, mPEG-RBCs (syngeneic, allogeneic and xenogeneic) show decreased immunogenicity. A number of studies show that, while PEG has low immunogenic potential, it does (under some circumstances) induce anti-PEG antibodies [32-35]. Typically, in animal models this has required the co-administration of Freund's Complete Adjuvant or coupling of PEG to highly immunogenic proteins [32,33]. More recently, studies have reported that a significant percentage of normal blood donors show evidence of anti-PEG antibodies [34,35]. However, most long-term studies on PEGylated human enzymes have failed to report any significant anti-PEG effects. Moreover, no antibody response (either $\operatorname{IgG}$ or $\operatorname{IgM}$ ) was noted in response to donor syngeneic mPEG-RBC in the murine transfusion model used in the present study. Indeed, repeated transfusion of syngeneic mPEG-RBCs showed no increased clearance in our murine model. More importantly, highly immunogenic allogeneic and xenogeneic cells demonstrated significantly decreased murine antibody responses in recipient mice.

In summary, global camouflage of RBC antigens by methoxypoly(ethylene glycol) derivatization dramatically attenuates both recognition of blood group antigens and the in vivo immunogenicity of antigenically disparate cells. Immunocamouflage arises from the biophysical effects of the grafting polymer, which both attenuates cell surface charge (hindering receptor-ligand interactions) and sterically impedes the approach and binding of antibodies and/or cells $[20,36]$. These data clearly suggest that PEGylation of non-ABO/RhD matched RBCs effectively reduces the risk of alloimmunization in chronically transfused patients. Ap- plication of this technology may be clinically useful in chronically transfused sickle and thalassemic patients because of the increased risk of alloimmunization faced by these patient populations.

This work was supported by grants from the Canadian Blood Services, Health Canada and the Canadian Blood Services-Canadian Institutes of Health Research (CBS-CIHR) Partnership Fund. We thank the Canada Foundation for Innovation and the Michael Smith Foundation for Health Research for infrastructure funding at the University of British Columbia Centre for Blood Research. The views expressed herein do not necessarily represent the view of the federal government of Canada.

1 Walker R H, Lin D T, Hartwick M B. Alloimmunization following blood transfusion. Arch Pathol Lab Med, 1989, 113: 254-261

2 Ambruso D R, Githens J H, Alcorn R, et al. Experience with donors matched for minor blood group antigens in patients with sickle cell anemia who are receiving chronic transfusion therapy. Transfusion, 1987, 27: 94-98

3 Fluit C R, Kunst V A, Drenthe-Schonk A M. Incidence of red cell antibodies after multiple blood transfusion. Transfusion, 1990, 30: 532-535

4 Vichinsky E P, Earles A, Johnson R A, et al. Alloimmunization in sickle cell anemia and transfusion of racially unmatched blood. New Eng J Med, 1990, 322: 1617-1621

5 Michail-Merianou V, Pamphili-Panousopoulou L, Piperi-Lowes L, et al. Alloimmunization to red cell antigens in thalassemia: Comparative study of usual versus better-match transfusion programmes. Vox Sang, 1987, 52: 95-98

6 McPherson M E, Anderson A R, Haight A E, et al. Transfusion management of sickle cell patients during bone marrow transplantation with matched sibling donor. Transfusion, 2009, 49: 1977-1986

7 Shulman I A. Prophylactic phenotype matching of donors for the transfusion of nonalloimmunized patients with sickle cell disease. Immunohematology, 2006, 22: 101-102

8 Castro O, Sandler S G, Houston-Yu P, et al. Predicting the effect of transfusing only phenotype-matched RBCs to patients with sickle cell disease: Theoretical and practical implications. Transfusion, 2002, 42: 684-690

9 Scott M D, Murad K L, Koumpouras F, et al. Chemical camouflage of antigenic determinants: Stealth erythrocytes. Proc Natl Acad Sci USA, 1997, 94: 7566-7571

10 Murad K L, Mahany K L, Brugnara C, et al. Structural and functional consequences of antigenic modulation of red blood cells with methoxypoly(ethylene glycol). Blood, 1999, 93: 2121-2127

11 Bradley A J, Test S T, Murad K L, et al. Interactions of IgM ABO antibodies and complement with methoxy-PEG-modified human RBCs. Transfusion, 2001, 41: 1225-1233

12 Bradley A J, Murad K L, Regan K L, et al. Biophysical consequences of linker chemistry and polymer size on stealth erythrocytes: Size does matter. Biochim Biophys Acta, 2002, 1561: 147-158

13 Bradley A J, Scott M D. Separation and purification of methoxypoly(ethylene glycol) grafted red blood cells via two-phase partitioning. J Chromatogr B Analyt Technol Biomed Life Sci, 2004, 807: 163-168

14 Bradley A J, Scott M D. Immune complex binding by immunocamouflaged [poly(ethylene glycol)-grafted] erythrocytes. Am J Hematol, 2007, 82: 970-975

15 Armstrong J K, Meiselman H J, Fisher T C. Covalent binding of poly(ethylene glycol) (PEG) to the surface of red blood cells inhibits aggregation and reduces low shear blood viscosity. Am J Hematol, 1997, 56: 26-28

16 Armstrong J K, Meiselman H J, Wenby R B, et al. Modulation of red blood cell aggregation and blood viscosity by the covalent attachment of Pluronic copolymers. Biorheology, 2001, 38: 239-247

17 Hortin G L, Lok H T, Huang S T. Progress toward preparation of 
universal donor red cells. Artif Cells Blood Substit Immobil Biotechnol, 1997, 25: 487-491

18 Tan Y, Qiu Y, Xu H, et al. Decreased immunorejection in unmatched blood transfusions by attachment of methoxypolyethylene glycol on human red blood cells and the effect on D antigen. Transfusion, 2006, 46: 2122-2127

19 Tan Y, Ji S, Li S, et al. Comparative assessment of normal and methoxypolyethylene glycol-modified murine red cells on swimming endurance and hippocampal injury in hypoxic mice. Transfusion, 2008, 48: 1954-1958

20 Le Y, Scott M D. Immunocamouflage: The biophysical basis of immunoprotection by grafted methoxypoly(ethylene glycol) (mpeg). Acta Biomater, 2010, 6: 2631-2641

21 Chen A M, Scott M D. Immunocamouflage: Prevention of transfusion-induced graft-versus-host disease via polymer grafting of donor cells. J Biomed Mater Res A, 2003, 67: 626-636

22 Chen A M, Scott M D. Comparative analysis of polymer and linker chemistries on the efficacy of immunocamouflage of murine leukocytes. Artif Cells Blood Substit Immobil Biotechnol, 2006, 34: 305-322

23 Reid M E, Lomas-Francis C. The Blood Group Antigen Facts Book. San Diego: Academic Press, 2003

24 Issitt P D. Race-related red cell alloantibody problems. Br J Biomed Sci, 1994, 51: 158-167

25 Beattie K M, Shafer A W. Broadening the base of a rare donor program by targeting minority populations. Transfusion, 1986, 26: 401-404

26 Thompson A A. Advances in the management of sickle cell disease. Pediatr Blood Cancer, 2006, 46: 533-539

27 Martin S. Fundamentals of Immunology for Blood Bankers. In: Har- mening DMMDS2708: F.A. Davis Company, 1994. 43-68

28 Hughes-Jones N C, Gardner B, Lincoln P J. Observation of the number of available c, D, and E antigen sites on red cells. Vox Sang, 1971, 21: $210-216$

29 Masouredis S P, Sudora E, Mahan L, et al. Quatitative immunoferritin microscopy of Fy-a, Fy-b, Jk-a, U, and Di-b antigen site numbers on human red cells. Blood, 1980, 56: 969-977

30 Skov F, Hughes-Jones N C. Observations on the number of available C antigen sites on red cells. Vox Sang, 1977, 33: 170-174

31 Murad K L, Gosselin E J, Eaton J W, et al. Stealth cells: Prevention of major histocompatibility complex class II-mediated T-cell activation by cell surface modification. Blood, 1999, 94: 2135-2141

32 Richter A W, Akerblom E. Antibodies against polyethylene glycol produced in animals by immunization with monomethoxy polyethylene glycol modified proteins. Int Arch Allergy Appl Immunol, 1983, 70: $124-131$

33 Richter A W, Akerblom E. Polyethylene glycol reactive antibodies in man: Titer distribution in allergic patients treated with monomethoxy polyethylene glycol modified allergens or placebo, and in healthy blood donors. Int Arch Allergy Appl Immunol, 1984, 74: 36-39

34 Armstrong J K, Hempel G, Koling S, et al. Antibody against poly(ethylene glycol) adversely affects PEG-asparaginase therapy in acute lymphoblastic leukemia patients. Cancer, 2007, 110: 103-111

35 Garratty G. Modulating the red cell membrane to produce universal/stealth donor red cells suitable for transfusion. Vox Sang, 2008, 94: 87-95

36 Sutton T C, Scott M D. The effect of grafted methoxypoly(ethylene glycol) chain length on the inhibition of respiratory syncytial virus (RSV) infection and proliferation. Biomaterials, 2010, 31: 4223-4230

Open Access This article is distributed under the terms of the Creative Commons Attribution License which permits any use, distribution, and reproduction in any medium, provided the original author(s) and source are credited. 